\title{
Chronic kidney disease and automatic reporting of estimated glomerular filtration rate: new developments and revised recommendations
}

David W Johnson MB BS (Hons), PhD, FRACP Nephrologist

Graham RD Jones MB BS, MSc(Med), DPhil Chemical Pathologist ${ }^{2}$

Timothy H Mathew MB BS, FRACP Medical Director

Marie J Ludlow BA(Hons), PhD, National Medical Projects Manager

Matthew P Doogue MB ChB, FRACP

Clinical Pharmacologist and Endocrinologist

Matthew D Jose MB BS, PhD, FRACP Honorary Fellow

Robyn G Langham MB BS, PhD, FRACP Nephrologist

Paul D Lawton MB BS, FRACP Nephrologist

Steven J McTaggart MB BS, PhD, FRACP Nephrologist

Michael J Peake MSc(Clin Biochem), FAIMS Medical Scientist

Kevan Polkinghorne MClinEpi, PhD, FRACP. Nephrologist ${ }^{10}$

Tim Usherwood MD, BS, FRACGP Professor of General Practice

Australasian Creatinine Consensus

Working Group

1Department of Nephrology, Princess Alexandra Hospital, Brisbane, QLD.

2 Department of Chemica Pathology, St Vincent's Hospital, Sydney, NSW.

3 Kidney Health Australia Adelaide, SA.

4 Department of Clinica Pharmacology, Flinders Medical Centre, Adelaide, SA.

5 Menzies Research Institute Tasmania University of Tasmania, Hobart, TAS.

6 Department of Nephrology, St Vincent's Hospital, Melbourne, VIC

An abridged version of this article appeared in the printed journal ollowing publication of the Australasian Creatinine Consensus Working Group's position statement in $2005^{1}$ and revised statement in $2007,^{2}$ a number of issues have arisen, including the development of the new Chronic Kidney Disease Epidemiology Collaboration (CKD-EPI) formula for estimated glomerular filtration rate (eGFR) determination, revisions to CKD staging, use of eGFR in other ethnic groups and population groups (such as paediatric patients and pregnant women), use of eGFR for drug dosing, progress of creatinine assay standardisation and use of enzymatic assays. Consequently, the Australasian Creatinine Consensus Working Group reconvened in 2010 to consider these issues. The group consisted of 12 representatives nominated by the parent bodies of this process (Royal College of Pathologists of Australasia, Australasian Association of Clinical Biochemists, Royal Australian College of General Practitioners, Australian and New Zealand Paediatric Nephrology Association, Australian and New Zealand Society of Nephrology, Australasian Society of Clinical and Experimental Pharmacologists and Toxicologists, and Kidney Health Australia). A larger stakeholder meeting of 31 participants was convened on 7 March 2011, with representatives from the aforementioned organisations as well as the Australian Practice Nurses Association, the Pharmaceutical Society of Australia, the Society of Obstetric Medicine of Australia and New Zealand, and the Kidney Health Australia National Consumer Council.

All evidence-based ${ }^{3,4}$ recommendations contained in this position statement are endorsed by the parent bodies of this process. It is also recommended that optimal screening for CKD and risk stratification of patients with CKD require careful evaluation of both eGFR and urinary albumin (see the recommendations of the Australasian Proteinuria Consensus Working Group). ${ }^{5}$

\section{Recommendations}

\section{Adoption of the CKD-EPI formula for calculating estimated glomerular filtration rate (eGFR)}

A significant limitation of the Modification of Diet in Renal Disease (MDRD) "175" formula is that it suffers from imprecision and systematic underestimation of GFR at higher values (above $60 \mathrm{~mL} / \mathrm{min} / 1.73 \mathrm{~m}^{2}$ ). Recently, the CKD Epidemiology Collaboration developed a new CKDEPI eGFR formula (Box) from a pooled dataset that included 10 studies and 8254 participants with and without known CKD (including participants with diabetes, potential kidney donors and transplant recipients). ${ }^{6}$ Validation of this formula in a separate external dataset of 3896 participants in 16 studies showed that the CKD-EPI for-

\section{- The publication of the Australasian Creatinine Consensus Working Group's position statements in 2005 and 2007 resulted in automatic reporting of estimated glomerular filtration rate (eGFR) with requests for serum creatinine concentration in adults, facilitated the unification of units of measurement for creatinine and eGFR, and promoted the standardisation of assays. New advancements and continuing debate led the Australasian Creatinine Consensus Working Group to reconvene in 2010.}

- The working group recommends that the method of calculating eGFR should be changed to the Chronic Kidney Disease Epidemiology Collaboration (CKD-EPI) formula, and that all laboratories should report eGFR values as a precise figure to at least $90 \mathrm{~mL} / \mathrm{min} / 1.73 \mathrm{~m}^{2}$.

- Age-related decision points for eGFR in adults are not recommended, as although an eGFR $<60 \mathrm{~mL} / \mathrm{min} / 1.73 \mathrm{~m}^{2}$ is very common in older people, it is nevertheless predictive of significantly increased risks of adverse clinical outcomes, and should not be considered a normal part of ageing.

- If using eGFR for drug dosing, body size should be considered, in addition to referring to the approved product information. For drugs with a narrow therapeutic index, therapeutic drug monitoring or a valid marker of drug effect should be used to individualise dosing.

- The CKD-EPI formula has been validated as a tool to estimate GFR in some populations of non-European ancestry living in Western countries. Pending publication of validation studies, the working group also recommends that Australasian laboratories continue to automatically report eGFR in Aboriginal and Torres Strait Islander peoples.

- The working group concluded that routine calculation of eGFR is not recommended in children and youth, or in pregnant women. Serum creatinine concentration (preferably using an enzymatic assay for paediatric patients) should remain as the standard test for kidney function in these populations.

mula retained the precision and accuracy of the MDRD formula at GFR $<60 \mathrm{~mL} / \mathrm{min} / 1.73 \mathrm{~m}^{2}$, and showed less bias and improved precision at GFR $>60 \mathrm{~mL} / \mathrm{min} / 1.73 \mathrm{~m}^{2} .7,8$ Subsequent epidemiological evaluations in North American $^{9}$ and Australian ${ }^{10}$ general population studies have shown that the CKD-EPI equation more appropriately categorises individuals with respect to long-term clinical risks of end-stage kidney disease, coronary heart disease, stroke and all-cause mortality than the MDRD equation. In particular, $1.9 \%$ of the Australian Diabetes, Obesity and Lifestyle Study ${ }^{10}$ population was reclassified as not having CKD, and reclassified individuals were predominantly younger women with a favourable cardiovascular risk profile and absence of significant albuminuria. The CKD Epidemiology Collaboration studies used 
* Coefficients for race are not included in these formulae. Note that these equations are only validated for use with isotope dilution mass spectrometry-traceable creatinine assays. eGFR = estimated glomerular filtration rate $\left(\mathrm{mL} / \mathrm{min} / 1.73 \mathrm{~m}^{2}\right)$. MDRD = modification of diet in renal disease. $\mathrm{SCr}=$ concentration of creatinine in serum. $\mathrm{CKD}-\mathrm{EPI}=\mathrm{Chronic}$ Kidney Disease Epidemiology Collaboration.

serum creatinine assays that were recalibrated, as required, to values aligned with the Roche enzymatic assay (Roche Diagnostics). As this assay has proven traceability to the isotope dilution mass spectrometry (IDMS) international reference method for creatinine measurement, the working group recommends that assays that are IDMS aligned (and otherwise suitable for the "175" MDRD equation) are suitable for routine use.

Changing from the MDRD formula to the CKD-EPI formula leads to higher eGFR values at normal or nearnormal levels of kidney function, particularly in younger individuals (<60 years). ${ }^{10}$ Among older people of European ancestry (men $>70$ years of age and women $>75$ years), median eGFR estimates are lower for the CKD-EPI formula compared with the MDRD, although the magnitude of these differences is small. ${ }^{11}$

It is important to recognise that improvements in the estimation of GFR achieved by the CKD-EPI formula do not ameliorate situations where creatinine-based GFRestimating equations are typically limited, including severe malnutrition or obesity, extremes of body size or age, exceptional dietary intake (vegetarian diet or creatine supplements), skeletal muscle disease, paraplegia, limb amputations or rapidly changing kidney function. ${ }^{12}$ Under such circumstances, GFR should be measured directly if there is doubt about whether kidney function is decreased. The use of the CKD-EPI formula also does not reduce the potential for creatinine assay interference by such substances as albumin, glucose, pyruvate, bilirubin, haemoglobin F and cephalosporins (especially cefpirome). ${ }^{13}$ Where interferences are considered to be likely, especially in neonates, the use of an enzymatic method may be preferable.

Recommendation: The method of calculating eGFR should be changed to the CKD-EPI formula. (Evidence level, 1C)

\section{Reporting limit for eGFR using the CKD-EPI formula}

Given that the CKD-EPI equation is more accurate (predominantly due to reduced bias) than the "175" MDRD equation at higher levels of kidney function, ${ }^{6-8}$ the working group considered increasing the recommended reporting limit for eGFR. Although no consensus could be reached on this issue, the working group concluded that all laboratories should report eGFR values as a precise figure to at least $90 \mathrm{~mL} / \mathrm{min} / 1.73 \mathrm{~m}^{2}$.

Recommendation: All laboratories should report eGFR values as a precise figure to at least $90 \mathrm{~mL} / \mathrm{min} / 1.73 \mathrm{~m}^{2}$. (Evidence level, 1C)
7 Division of Medicine, Royal Darwin Hospital, Darwin, NT.

8 Queensland Child and Adolescent Renal Service, Royal Children's Hospital, Brisbane, QLD.

9 Department of Medical Biochemistry, Flinders Medical Centre, Adelaide, SA.

10 Department of Nephrology, Monash Medical Centre, Melbourne, VIC.

11 Department of General Practice, Sydney Medical School Westmead, Sydney, NSW.

marie.ludlow@ kidney.org.au

doi: 10.5694/mjall.11329

.

\section{Serum creatinine assay performance in adult populations}

In Australia and New Zealand, routine use of serum creatinine assays that are IDMS traceable has significantly improved the between-laboratory difference in creatinine results. However, there are still differences in performance with regard to assay precision, variation from this standard, and interferences due to limitations in the analytical specificity of assays. To guide laboratories in selecting and running creatinine assays, it is necessary to have a quality standard for assay performance. The bias criterion of $\pm 5 \%$ is the minimal standard based on a biological variation approach as proposed by the National Kidney Disease Education Program in the United States. ${ }^{14}$ A local evaluation of the effect of a $5 \%$ bias indicated that about $5 \%$ of general practice patients over 50 years of age having eGFR calculated would have their classification of stage of CKD changed at the level of $60 \mathrm{~mL} / \mathrm{min} / 1.73 \mathrm{~m}^{2}$ (GRD J, unpublished data, 2011). This criterion is a challenge for laboratories to meet, but is achievable with modern assays. ${ }^{15}$

The effect of assay imprecision has relatively little effect on classification according to eGFR but can have a significant effect when monitoring serum creatinine concentration or eGFR in an individual. A coefficient of variation (CV) of $6.0 \%$ describes the average within-subject biological variation for serum creatinine $(\mathrm{CVi}),{ }^{16}$ so an assay with a CV below $4.5 \%$ ( $0.75 \%$ of $\mathrm{CVi})$ contributes less than an additional $25 \%$ to the total result variation. Better precision is preferred, but the benefits in clinical decision making are less marked. Most Australian and New Zealand laboratories can meet this specification at the creatinine concentrations found in adults.

Recommendation: The performance of serum creatinine assays should achieve a bias $\pm 5 \%$ (ie, $5 \mu \mathrm{mol} / \mathrm{L}$ at a value of $100 \mu \mathrm{mol} / \mathrm{L}$ ) and a long-term within-laboratory coefficient of variation (CV) less than $4 \%$ for the measurement of serum creatinine in adults. (Evidence level, 2C)

\section{Age-related reference intervals for eGFR in adults}

The Chronic Kidney Disease Prognosis Consortium recently published findings of collaborative meta-analyses of data from general ${ }^{17,18}$ and high-risk ${ }^{19}$ populations, and populations with kidney disease ${ }^{20}$ (45 cohorts in total, comprising 1555332 participants, summarised in Levey et $\left.\mathrm{al}^{21}\right)$. These data showed that eGFR $<60 \mathrm{~mL} / \mathrm{min} / 1.73 \mathrm{~m}^{2}$ was associated with increased risks of all-cause mortality, cardiovascular mortality, end-stage kidney disease, acute kidney injury and progression of CKD without consistent age interactions. In particular, for the controversial cate- 
gory of eGFR $45-59 \mathrm{~mL} / \mathrm{min} / 1.73 \mathrm{~m}^{2}$ with normal albuminuria, the relative hazards of all outcomes except allcause mortality were similar above and below the age of 65 years in the general population cohorts. ${ }^{17}$ These observations are not consistent with the interpretation that decreased GFR with ageing is "normal" or "physiological". Consequently, the working group concluded that agerelated decision points for eGFR are not recommended in adults.

Recommendation: Age-related decision points for eGFR are not recommended in adults. (Evidence level, 1C)

\section{The use of eGFR for adjusting drug dosing in patients with reduced kidney function}

Renal drug clearance, which is usually proportional to GFR, is an important factor to consider when selecting a drug dose for a patient with CKD. Most official recommendations for drug dosing in kidney impairment are traceable to the manufacturers' data by measured GFR or creatinine clearance estimated by the Cockcroft-Gault formula. ${ }^{22,23}$ Few studies have been conducted directly linking dosing according to eGFR with pharmacokinetic or clinical outcomes. However, eGFR provides a valid estimate of GFR and is widely available on laboratory reports. The units of eGFR are $\mathrm{mL} / \mathrm{min} / 1.73 \mathrm{~m}^{2}$ whereas the units of drug clearance are $\mathrm{mL} / \mathrm{min} .^{24}$ To avoid overdosing small patients or underdosing large patients, eGFR should be adjusted for patient size. In CKD, factors other than renal drug clearance can also alter drug effects. Thus, for drugs with a narrow therapeutic index, drug effects (desired and adverse) or drug concentrations should be monitored. ${ }^{25}$ Detailed advice on drug dosing is outside the scope of this document. $^{26}$

Recommendation: Dose reduction of some drugs is recommended for patients with reduced kidney function. Both eGFR $\left(\mathrm{mL} / \mathrm{min} / 1.73 \mathrm{~m}^{2}\right)$ and estimated creatinine clearance $(\mathrm{mL} / \mathrm{min})$ provide an estimate of relative renal drug clearance. If using eGFR for drug dosing, body size should be considered, in addition to referring to the approved product information. For drugs with a narrow therapeutic index, therapeutic drug monitoring or a valid marker of drug effect should be used to individualise dosing. (Evidence level, 1C)

\section{The use of eGFR in various ethnic populations}

Both the MDRD and CKD-EPI formulae contain a factor to be applied to African Americans, raising the possibility that other variations in the formulae may be required for optimal performance in different ethnic groups. Indeed, several studies have shown that performance of the MDRD equation in China ${ }^{27}$ and Japan ${ }^{28}$ improved when population-specific coefficients were introduced. A recent study evaluated a GFR-estimating equation that incorporated a four-level race variable (black, Asian, Native American and Hispanic, and white and other) against CKD-EPI eGFR (incorporating a two-level race variable, African American or not) in a validation cohort of 4014 patients from 17 studies from the United States and Europe, as well as in 1022 patients from China, Japan and South Africa. ${ }^{29}$ The CKD-EPI equation showed minimal bias in black, Native American and Hispanic, and white and other cohorts. The four-level ethnicity equation improved bias in Asian patients, but showed large bias in Japanese and South African patients, and such heterogeneity in performance among multiple ethnicities precluded its use. The study showed that the CKD-EPI equation (with a twolevel race variable) could be successfully applied across a broad range of racial and ethnic groups living in Western countries, with the understanding that there is likely to be some variation in the accuracy of GFR estimates among and within these groups, based on factors associated with creatinine generation (principally diet and muscle mass).

A specific need in Australia and New Zealand is for GFRestimating equations (including CKD-EPI) to be validated in Aboriginal and Torres Strait Islander peoples and Maori and Pacific Islander peoples, so that a firm basis for their use can be established. Until this evidence is available, it appears clinically appropriate for CKD-EPI eGFR to be calculated and used prudently in these ethnic groups, using the non-African American formulae. A validation study in Aboriginal and Torres Strait Islander peoples comparing eGFR to measured GFR is currently underway. ${ }^{30}$

Recommendation 6a: The CKD-EPI formula is a useful tool to estimate GFR in all people, including various ethnic populations.

Recommendation 6b: The CKD-EPI formula has been validated as a tool to estimate GFR in some nonEuropean populations, including South-East Asian, African, Indian and Chinese individuals living in Western countries.

Recommendation 6c: The different methods to estimate GFR from serum creatinine concentration have not been validated in Indigenous Australians, although these studies are currently underway. (Evidence level, 2C)

\section{The use of eGFR in pregnancy}

Significant physiological changes occur in normal pregnancy, some of which affect kidney function. However, studies of kidney function in normal pregnancy are limited, and may be non-existent in some ethnic populations. Kidney function changes throughout gestation, but becomes particularly important in medical disorders, such as pre-eclampsia, when reduced function may occur.

Validation studies of eGFR in pregnant women have not been performed. A 24-hour creatinine clearance, when the collection is complete, does provide a valid estimate of kidney function. Reference ranges for serum creatinine are available for the entire 40 -week gestation, although the reference range will vary frequently, up to every 4 weeks. ${ }^{31}$ Additional studies to validate the use of eGFR as a measure of kidney function in pregnant women, particularly in ethnic subgroups, would be welcomed.

Recommendation: The validity of eGFR in pregnancy is not known. Serum creatinine concentration should be maintained as the standard test for kidney function in pregnant women. (Evidence level, 1C)

\section{Measurement of serum creatinine concentration and} calculation of eGFR in paediatric populations

There are limited and conflicting data on the value of routine screening for CKD in children and youth (individ- 
uals aged less than 18 years). While a number of countries (predominantly within Asia) have instigated school-based screening programs, current publications have not convincingly shown that that early detection of kidney disorders in children will lead to a reduction in clinically significant outcomes. ${ }^{32}$ As such, the cost-effectiveness of such an approach has been questioned. ${ }^{33}$ Moreover, calculation of eGFR in children is logistically problematic because the most commonly used equation, the Schwartz formula, requires height data, which are not routinely measured at the time of blood collection. Thus, the working group does not recommend routine reporting of eGFR in children.

Creatinine production is a function of muscle mass and varies considerably throughout infancy and childhood as a result of growth and development. Serum creatinine concentration in normal infants and children increases with age and is slightly higher at any age in males than females. ${ }^{34}$ Normative values, obtained from studies that used creatinine assays that were traceable to the IDMS reference method have been published ${ }^{35}$ and are recommended for more accurate reporting of kidney function in children and adolescents. Although enzymatic and nonenzymatic methods are considered acceptable for measurement of creatinine in paediatric patients, enzymatic methods are generally preferred, particularly in young infants. Enzymatic methods are not affected by other substances (albumin, IgG and haemoglobin F) that are known to interfere with Jaffe creatinine assays and may lead to clinically important inaccuracies in the measurement of serum creatinine concentration. ${ }^{36}$ A detailed comparison of interferences in Jaffe and enzymatic creatinine assays has recently been published. ${ }^{37}$

Recommendation: The use of an enzymatic assay is recommended for the measurement of serum creatinine concentration in children and youth (individuals aged less than 18 years). Other serum creatinine assays that achieve a bias $<10 \%$ at low serum creatinine values (ie, $5 \mu \mathrm{mol} / \mathrm{L}$ at a value of $50 \mu \mathrm{mol} / \mathrm{L}$ ) and are not compromised by variations in albumin, bilirubin and haemoglobin F (neonates) would be satisfactory alternatives. Routine calculation of eGFR is not recommended in children and youth. Age-appropriate reference values for serum creatinine concentration should be reported for individuals up to 18 years of age. (Evidence level, 1C)

\section{Conclusion}

The available evidence indicates that introduction of automatic reporting of eGFR each time a test for serum creatinine concentration is requested has increased the awareness of significant kidney dysfunction in clinical practice, augmented the detection of patients with CKD in the community and enhanced the quantity of appropriate referrals to specialist renal services. ${ }^{38}$ It has also led to improvements in the accuracy and standardisation of laboratory measurement of creatinine and a reduction in the variability previously seen in Australasia and overseas. Progressive refinements in GFR estimating equations, from Cockcroft-Gault to "186" MDRD to "175" MDRD to CKD-EPI eGFR, have resulted in improved accuracy, par- ticularly at normal and near-normal levels of kidney function, and in better kidney and cardiovascular risk prediction. However, clinicians should also be aware that there is now overwhelming evidence that optimal detection and subsequent risk stratification of CKD patients requires simultaneous consideration of both eGFR and urinary albumin, rather than eGFR alone.

Competing interests: David Johnson has received consulting fees or honoraria from Amgen, Roche, Shire, Genzyme, Boehringer-Ingelheim, Baxter Healthcare and Fresenius Medical Care; support for travel to meetings from Baxter, Amgen, Roche and Shire; and payment for development of educational presentations from the Australian College of Rural and Remote Medicine and the Northern Territory Division of General Practice.

Graham Jones has received research support from Roche Diagnostics and support for travel to meetings from Roche Diagnostics and Bio-Rad.

Steven McTaggart has received funding for travel from Amgen, Roche and Janssen-Cilag.

Provenance: Not commissioned; externally peer reviewed.

1 Mathew TH; Australasian Creatinine Consensus Working Group. Chronic kidney disease and automatic reporting of estimated glomerular filtration rate: a position statement. Med J Aust 2005; 183: 138-141.

2 Mathew TH, Johnson DW, Jones GRD; on behalf of the Australasian Creatinine Consensus Working Group. Chronic kidney disease and automatic reporting of estimated glomerular filtration rate: revised recommendations. Med J Aust 2007; 187: 459-463.

3 Atkins D, Best D, Briss PA, et al; GRADE Working Group. Grading quality of evidence and strength of recommendations. BMJ 2004; 328: 1490.

4 Uhlig K, Macleod A, Craig J, et al. Grading evidence and recommendations for clinical practice guidelines in nephrology. A position statement from Kidney Disease: Improving Global Outcomes (KDIGO). Kidney Int 2006; 70: 20582065.

5 Johnson DW, Jones GRD, Mathew TH, et al; Australasian Proteinuria Consensus Working Group. Chronic kidney disease and measurement of albuminuria or proteinuria: a position statement. Med J Aust 2012; 197: 222-223.

6 Levey AS, Stevens LA, Schmid CH, et al; CKD-EPI (Chronic Kidney Disease Epidemiology Collaboration). A new equation to estimate glomerular filtration rate. Ann Intern Med 2009; 150: 604-612.

7 Levey AS, Stevens LA. Estimating GFR using the CKD Epidemiology Collaboration (CKD-EPI) creatinine equation: more accurate GFR estimates, lower CKD prevalence estimates, and better risk predictions. Am J Kidney Dis 2010; 55: 622-627.

8 Stevens LA, Schmid CH, Greene T, et al. Comparative performance of the CKD Epidemiology Collaboration (CKD-EPI) and the Modification of Diet in Renal Disease (MDRD) study equations for estimating GFR levels above $60 \mathrm{~mL} / \mathrm{min}$ / $1.73 \mathrm{~m}^{2}$. Am J Kidney Dis 2010; 56: 486-495.

9 Matsushita K, Selvin E, Bash LD, et al. Risk implications of the new CKD Epidemiology Collaboration (CKD-EPI) equation compared with the MDRD Study equation for estimated GFR: the Atherosclerosis Risk in Communities (ARIC) Study. Am J Kidney Dis 2010; 55: 648-659.

10 White SL, Polkinghorne KR, Atkins RC, Chadban SJ. Comparison of the prevalence and mortality risk of CKD in Australia using the CKD Epidemiology Collaboration (CKD-EPI) and Modification of Diet in Renal Disease (MDRD) Study GFR estimating equations: the AusDiab (Australian Diabetes, Obesity and Lifestyle) Study. Am J Kidney Dis 2010; 55: 660-670.

11 van den Brand JA, van Boekel GA, Willems HL, et al. Introduction of the CKDEPI equation to estimate glomerular filtration rate in a Caucasian population. Nephrol Dial Transplant 2011; 26: 3176-3181. doi: 10.1093/ndt/gfr003.

12 Johnson D; Caring for Australians with Renal Impairment. The CARI guidelines. Evaluation of renal function. Nephrology (Carlton) 2005; 10 Suppl 4: S133S176.

13 Peake M, Whiting M. Measurement of serum creatinine - current status and future goals. Clin Biochem Rev 2006; 27: 173-184.

14 Myers GL, Miller GW, Coresh J, et al. Recommendations for improving serum creatinine measurement: a report from the Laboratory Working Group of the National Kidney Disease Education Program. Clin Chem 2006; 52: 5-18.

15 Piéroni L, Delanaye P, Boutten A, et al; Société Française de Biologie Clinique. A multicentric evaluation of IDMS-traceable creatinine enzymatic assays. Clin Chim Acta 2011; 412: 2070-2075.

16 Westgard QC. Desirable biological variation database specifications. http:// www.westgard.com/biodatabase-2012-update.htm (accessed Aug 2012).

17 Chronic Kidney Disease Prognosis Consortium. Association of estimated glomerular filtration rate and albuminuria with all-cause and cardiovascular mortality in general population cohorts: a collaborative meta-analysis. Lancet 2010; 375: 2073-2081.

18 Gansevoort RT, Matsushita K, van der Velde M, et al; Chronic Kidney Disease Prognosis Consortium. Lower estimated GFR and higher albuminuria are associated with adverse kidney outcomes. A collaborative meta-analysis of general and high-risk population cohorts. Kidney Int 2011; 80: 93-104. doi: $10.1038 / \mathrm{ki} .2010 .531$. 
19 van der Velde M, Matsushita K, Coresh J, et al. Lower estimated glomerular filtration rate and higher albuminuria are associated with all-cause and cardiovascular mortality. A collaborative meta-analysis of high-risk population cohorts. Kidney Int 2011; 79: 1341-1352.

20 Astor BC, Matsushita K, Gansevoort RT, et al. Lower estimated glomerular filtration rate and higher albuminuria are associated with mortality and endstage renal disease. A collaborative meta-analysis of kidney disease population cohorts. Kidney Int 2011; 79: 1331-1340. doi: 10.1038/ki.2010.550.

21 Levey AS, de Jong PE, Coresh J, et al. The definition, classification, and prognosis of chronic kidney disease: a KDIGO Controversies Conference report. Kidney Int 2010; 80: 17-28. doi: 10.1038/ki.2010.483.

22 US Department of Health and Human Services Food and Drug Administration. Guidance for industry: pharmacokinetics in patients with impaired renal function - study design, data analysis, and impact on dosing and labeling. Rockville, MD: Center for Drug Evaluation and Research and Center for Biologics Evaluation and Research, 1998. http://www.fda.gov/downloads/ Drugs/GuidanceComplianceRegulatoryInformation/Guidances/ ucm072127.pdf (accessed Apr 2012).

23 Committee for Medicinal Products for Human Use (CHMP). Note for guidance on the evaluation of the pharmacokinetics of medicinal products in patients with impaired renal function. London, UK: European Medicines Agency, 2004. http://www.ema.europa.eu/docs/en_GB/document_library/Scientific guideline/2009/09/WC500003123.pdf (accessed Apr 2012).

24 Jones GR. Estimating renal function for drug dosing decisions. Clin Biochem Rev 2011; 32: 81-88.

25 Doogue MP, Polasek TM. Drug dosing in renal disease. Clin Biochem Rev 2011; 32: 69-73.

26 Cervelli MJ, editor. The renal drug reference guide. Adelaide, SA: Kidney Health Australia, 2007.

$27 \mathrm{Ma} \mathrm{YC,} \mathrm{Zuo} \mathrm{L,} \mathrm{Chen} \mathrm{JH,} \mathrm{et} \mathrm{al.} \mathrm{Modified} \mathrm{glomerular} \mathrm{filtration} \mathrm{rate} \mathrm{estimating}$ equation for Chinese patients with chronic kidney disease. J Am Soc Nephrol 2006; 17: 2937-2944.
28 Imai E, Horio M, Nitta K, et al. Estimation of glomerular filtration rate by the MDRD study equation modified for Japanese patients with chronic kidney disease. Clin Exp Nephrol 2007; 11: 41-50.

29 Stevens LA, Claybon MA, Schmid CH, et al. Evaluation of the Chronic Kidney Disease Epidemiology Collaboration equation for estimating the glomerular filtration rate in multiple ethnicities. Kidney Int 2011; 79: 555-562.

30 Maple-Brown LJ, Lawton PD, Hughes JT, et al. Study Protocol - accurate assessment of kidney function in Indigenous Australians: aims and methods of the eGFR Study. BMC Public Health 2010; 10: 80.

31 Girling JC. Re-evaluation of plasma creatinine concentration in normal pregnancy. J Obstet Gynaecol 2000; 20: 128-131.

32 Hogg RJ. Screening for CKD in children: a global controversy. Clin J Am Soc Nephrol 2009; 4: 509-515.

33 Kaplan RE, Springate JE, Feld LG. Screening dipstick urinalysis: a time to change. Pediatrics 1997; 100: 919-921.

34 Rhodin MM, Anderson BJ, Peters AM, et al. Human renal function maturation: a quantitative description using weight and postmenstrual age. Pediatr Nephrol 2009; 24: 67-76.

35 Ceriotti F, Boyd JC, Klein G, et al; IFCC Committee on Reference Intervals and Decision Limits. Reference intervals for serum creatinine concentrations: assessment of available data for global application. Clin Chem 2008; 54: 559566.

36 Cobbaert CM, Baadenhuijsen H, Weykamp CW. Prime time for enzymatic creatinine methods in pediatrics. Clin Chem 2009; 55: 549-558.

37 Greenberg N, Roberts WL, Bachmann LM, et al. Specificity characteristics of 7 commercial creatinine measurement procedures by enzymatic and Jaffe method principles. Clin Chem 2012; 58: 391-401.

38 Noble E, Johnson DW, Gray N, et al. The impact of automated eGFR reporting and education on nephrology service referrals. Nephrol Dial Transplant 2008; 23: $3845-3850$. 